\title{
Absorption of electrolytes from the colon in cases of ulcerative colitis and in control subjects
}

\author{
D. GOOPTU, S. C. TRUELOVE, AND G. T. WARNER \\ From the Nuffield Department of Clinical Medicine, Radcliffe Infirmary, \\ Oxford
}

Although ulcerative colitis was described as a disease entity by Wilks in 1874, it was not until 1950 that electrolyte depletion was recognized as a major complication. The deficiency may be so severe as to cause death. Accounts of the losses of the monovalent cations, sodium and potassium, have been published by Posey and Bargen (1950), Lubran and McAllen (1951), and by Smiddy, Gregory, Smith, and Goligher (1960). The divalent cations, magnesium and calcium, are also lost from the body (Posey and Bargen, 1950; Thorén, 1962 and 1963).

The present study had two main objects. The first was to see the extent to which the normal human colon could absorb sodium, potassium, magnesium, and calcium ions. The second was to look for differences in absorption between a healthy colon and one affected with ulcerative colitis. As secondary investigations the rates of clearance of sodium and potassium from the blood were measured and an attempt was also made to measure the loss of potassium from the blood into the colonic lumen.

\section{MATERIALS}

Radioactive isotopes were used for these observations. Chemically pure ${ }^{24} \mathrm{Na},{ }^{42} \mathrm{~K},{ }^{47} \mathrm{Ca}$, and ${ }^{28} \mathrm{Mg}$ were supplied by the Radiochemical Centre, Amersham, as sterile solutions of the chloride salt. These particular isotopes were chosen because of their short half-lives.

Before embarking on the use of the radioactive substances, particulars of the isotopes to be studied, the dosages envisaged, and the criteria proposed for the selection of the human volunteers were furnished to the Medical Research Council. The subjects were selected and investigated in accordance with the directions of the Council's Advisory Panel for the Allocation of Radioactive Isotopes for Clinical Use. The nature of the experiments and the personal hazards involved were fully explained to the volunteers and their consent obtained. No volunteer was used more than once.

One group of subjects suffered from ulcerative colitis. At the time of experiment, some of these subjects were experiencing an acute attack of the disease, some were in a mild relapse of chronic disease, and some were in complete clinical remission. For comparison volunteers were recruited from patients admitted to hospital for other reasons and whose colons were in a healthy state. At the time of experiment these control subjects were convalescent from their illness and were in good general condition. The control subjects were adults and, in the case of women, were either towards the end of their reproductive period of life or beyond it.

\section{METHODS}

ABSORPTION EXPERIMENTS The control subjects had the colon washed out with water about one hour beforehand to reduce the faecal contents as much as possible. This was not considered necessary in the patients with ulcerative colitis in an active phase. All investigations were undertaken in the ward with the subjects in bed; the patients with ulcerative colitis were not being given any medical treatment at the time.

The radioactive test solutions were made up to contain $20 \mu \mathrm{c}$ in a total volume of $100 \mathrm{ml}$ isotonic with blood plasma, the diluent having the same chemical composition as the original isotope solution. For example, radioactive sodium chloride solution was made up with normal saline. The measured volume of the test solution was carefully run from a burette into an empty disposable plastic enema bag fitted with a $7 \mathrm{~cm}$ plastic nozzle from which it could be instilled into the subject's rectum. An aliquot of the original solution containing $0.01 \mu \mathrm{c}$ was kept as a standard for subsequent measurements.

Before the instillation of the test solution a sample of venous blood was withdrawn and stored in a chromicacid-cleaned heparinized container. The subject was then asked to lie on his left side and the nozzle of the plastic enema bag was passed for its whole length through the anal canal into the rectum. The bag was emptied by squeezing and rolling up. When all the fluid had run in the nozzle was withdrawn and the subject was turned prone to facilitate the spread of the solution along the colon. After instillation further samples of venous blood were withdrawn into heparinized containers, the first after 10 minutes and the next four at half-hourly intervals. Thereafter three samples were taken at intervals of one hour until the experiment was terminated at the end of five hours. 
CleARANCE EXPERIMENTS Potassium chloride solution containing $20 \mu \mathrm{c}$ of ${ }^{42} \mathrm{~K}$ was diluted with sterile distilled water to a volume of $5 \mathrm{ml}$ and was injected intravenously into one arm of the subject. A total of nine samples of venous blood were taken from the other arm, one immediately before the injection, another 10 minutes after it, and then at 30-and 60-minute intervals as in the absorption studies. An aliquot of the test solution was kept as before and counted as detailed in the next section.

To explore the substantial loss of potassium that occurs from the intestinal tract in an acute stage of ulcerative colitis, ${ }^{42} \mathrm{~K}$ as chloride was injected intravenously and the faeces were collected to measure their radioactivity. All faeces passed in six hours after the injection were collected and were counted in a plastic phosphor well scintillation counter (Warner and Oliver, 1962).

In order to find out whether there was any abnormality in the rate of clearance of injected sodium and potassium chlorides during the first $\mathbf{1 0}$ or $\mathbf{1 2}$ minutes after injection in ulcerative colitis a further group of subjects was examined. Patients and controls were given either $10 \mu \mathrm{c}$ of ${ }^{24} \mathrm{Na}$ or $20 \mu \mathrm{c}$ of ${ }^{42} \mathrm{~K}$ by intravenous injection in one arm and samples of venous blood were taken from the other arm at one-minute intervals.

MEASUREMENT TECHNIQUE The standard portion of isotope solution containing $0.01 \mu \mathrm{c}$ retained at the start of each absorption experiment was made up to a volume of $4 \mathrm{ml}$ and its scintillation count rates were measured in a sodium iodide crystal well counter. Four $\mathrm{ml}$ of venous blood, taken by a pipette from each sample, was then counted. The scintillation count rates obtained from each blood sample were expressed as a percentage of that for the standard. To take into account the physical decay of the radioactive isotope used, the count rates of the standard solution were obtained again in the middle and at the end of each experiment. When the whole series of blood samples had been counted relative activities were plotted graphically against time.

\section{RESULTS}

SODIUM ABSORPTION EXPERIMENT The absorption of ${ }^{24} \mathrm{Na}$ was studied in eight patients with ulcerative colitis in an active stage and in four patients with ulcerative colitis in remission. The results from the eight patients with active colitis are comparedwith the rates observed in 10 control subjects in the composite graph (Fig.1).

In the normal control subjects, the curve representing the amount of ${ }^{24} \mathrm{Na}$ in the plasma rose steadily during a period of about three hours. It then levelled out to form a plateau which persisted until the end of the five-hour experimental period.

When the mean values of the count rates obtained from the patients with active ulcerative colitis are compared with those obtained from the control subjects, the rise of the curve during the first 40 minutes is seen to be much steeper (Fig. 2). Also, while both groups tend to reach a peak after three hours, the count rates in ulcerative colitis are almost twice as

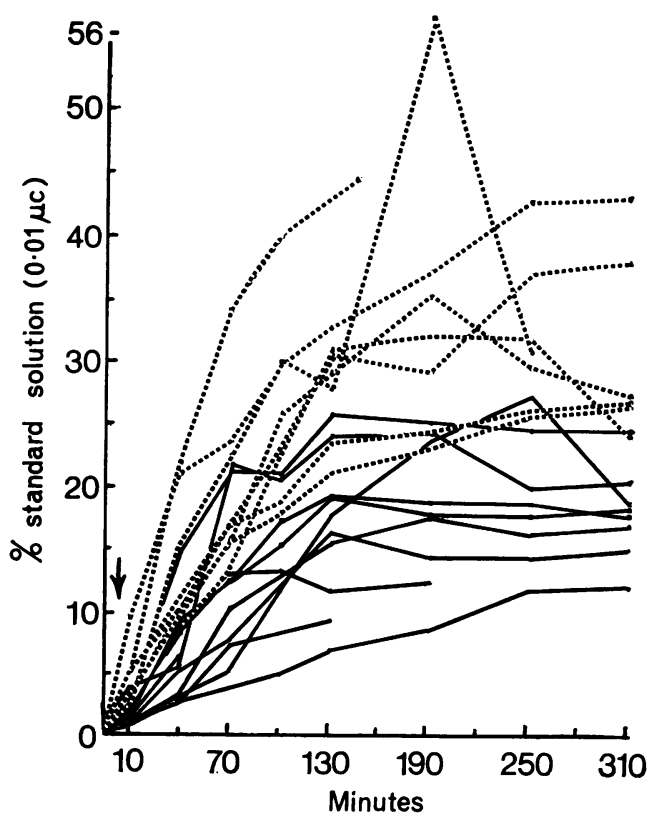

FIG. 1. Levels of radioactivity in the blood after instillation of ${ }^{24} \mathrm{Na}$ into the colon in ulcerative colitis patients (dotted line) and in normal subjects (continuous line).

high as in subjects with a normal colon. The difference is significant $(P<0.05)$.

The four patients with ulcerative colitis in remission were clinically normal, with a normal appearance of the rectosigmoid mucosa on sigmoidoscopy, and with no inflammation shown in rectal biopsy specimens examined histologically. Their scintillation count rates were found to lie between those of the other two groups but were more closely related to those obtained from the patients with active colitis.

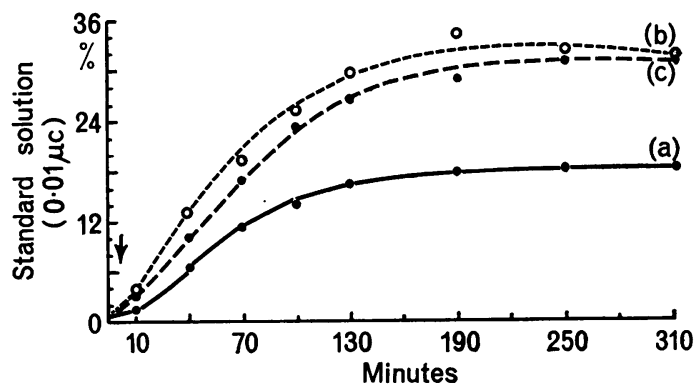

FIG. 2. Comparison of radioactivity in the blood in normal subjects (a), patients with active ulcerative colitis (b), and patients with ulcerative colitis in clinical remission (c) after instillation of ${ }^{24} \mathrm{Na}$ into the colon (mean values for each group of subjects). 


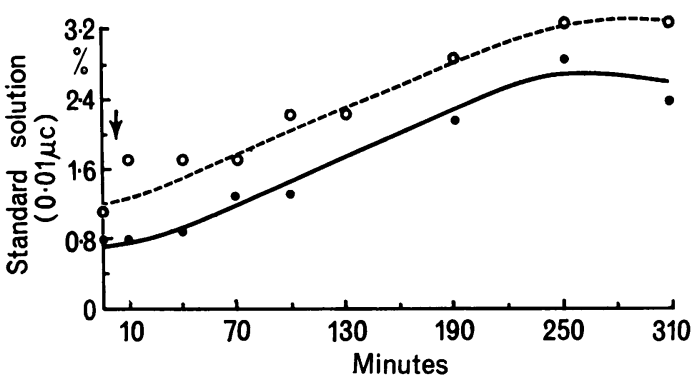

FIG. 3. Comparison of potassium outflux from the colon both in normal subjects (continuous line) and in ulcerative colitis patients (dotted line). There is no significant difference in the rate or degree of absorption between the two groups.

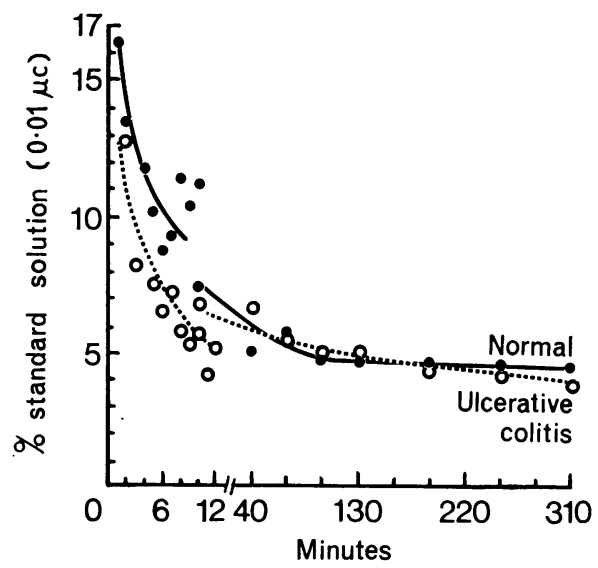

FIG. 4. Levels of radioactivity in the blood after intravenous injection of ${ }^{42} \mathrm{~K}$. The clearance of potassium is similar in ulcerative colitis patients and control subjects.

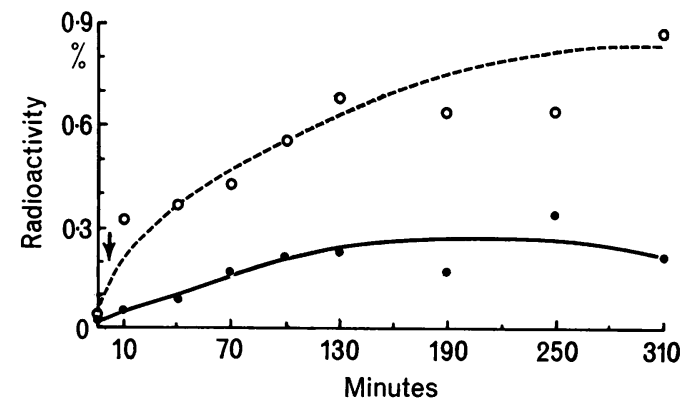

FIG. 5. Levels of radioactivity in the blood after instillation of ${ }^{28} \mathrm{Mg}$ into the colon. Higher levels are shown in ulcerative colitis patients (dotted line) than in normal subjects (continuous line).
Sodium clearance For this part of the experiment, two normal subjects and two patients with active ulcerative colitis were examined. A standard quantity of ${ }^{24} \mathrm{Na}$ as sodium chloride solution was injected intravenously into these subjects. After this injection, samples of venous blood were taken at one-minute intervals for the next 12 minutes. The level of radioactivity in the blood was similar in the subjects with ulcerative colitis and control subjects thoughout this period of time. In other words, there was no evidence of impaired clearance of ${ }^{24} \mathrm{Na}$ from the blood in ulcerative colitis, which was theoretically possible either because of a reduced body pool of sodium or because of impaired renal function.

POTASSIUM ABSORPTION EXPERIMENT The total number of patients in this series was 11 , six being control subjects and five having ulcerative colitis in an active phase. Figures for the relative radioactivity of the blood samples at the different intervals after the instillation are expressed graphically in Figure 3. They show that there is a small amount of potassium absorption from the normal colon and that the blood concentration of ${ }^{42} \mathrm{~K}$ rises to a peak after four hours. Colonic absorption of ${ }^{42} \mathrm{~K}$ in ulcerative colitis follows a similar pattern and there is no significant difference in the rate or degree of absorption between the two groups.

Potassium clearance Three patients with active ulcerative colitis and four other subjects acting as normal controls were used for the experiments lasting five hours, and a further four subjects, two from each group, took part in the short-term tests over a period of 12 minutes.

Mean values for the scintillation counts of both series from the two groups are shown in Figure 4. They show no significant differences between the ulcerative colitis patients and the control subjects.

There was no demonstrable increase of radioactivity in the faeces either of ulcerative colitis patients or of the control group after injection of $20 \mu \mathrm{c}$ of ${ }^{42} \mathrm{~K}$, presumably because of the very high dilution of the injected ${ }^{42} \mathrm{~K}$ in the body's potassium pool.

MAGNESIUM ABSORPTION EXPERIMENT The total number of subjects in this study was four, two of whom were control subjects. The small size of these groups is regrettable but was dictated by the high cost of the radioactive isotope ( $£ 150$ per batch) in relation to the research funds available.

As seen with sodium the magnesium absorption figures showed a wide range of differences between individuals. Nevertheless, in comparison with the normal subjects, the absorption of ${ }^{28} \mathrm{Mg}$ in both 


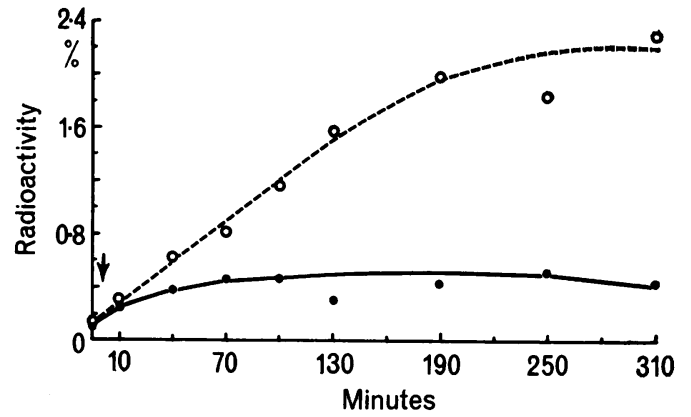

FIG. 6. Radioactivity levels in the blood after instillation of ${ }^{47} \mathrm{Ca}$ into the colon. Higher levels are shown in ulcerative colitis patients (dotted line) than in normal subjects (continuous line).

ulcerative colitis patients was relatively high. The mean percentage figures for blood radioactivity after instillation of ${ }^{28} \mathrm{Mg}$ are shown in Figure 5.

CALCIUM ABSORPTION EXPERIMENT Instillation of ${ }^{47} \mathrm{Ca}$ as calcium chloride was carried out in a total of four subjects, two with ulcerative colitis and two controls. As in the magnesium studies the numbers were limited by considerations of cost.

Once again, the percentage activity figures for each blood sample showed a wide range of individual variation. One member of the control group produced values which were only slightly above the level of background count. At the other extreme, one of the ulcerative colitis patients was found to absorb large amounts of ${ }^{47} \mathrm{Ca}$ progressively over the whole five-hour period. The mean values are given in Figure 6.

\section{DISCUSSION}

Our knowledge of the role of the human colon in the net flux of electrolytes and other substances is still uncertain. It is known that the colon absorbs sodium chloride from its contents and the present observations confirm this fact. At the same time there are contradictory opinions whether other electrolytes, such as potassium, calcium, and magnesium, can be taken up by the normal colon. The results presented here indicate that they can.

In ulcerative colitis there are drastic changes in the colonic mucosa and profound metabolic disturbances due to deficiency of electrolytes may also occur. Lubran and McAllen (1951) and Smiddy et al (1960) concluded that the colonic mucosa absorbed less in an inflamed state although they had no direct evidence that the depletion of electrolytes was due to a defect of colonic absorption. Duthie, Watts, De Dombal, and Goligher (1964) have recorded a diminished absorption of sodium and potassium from the colon in some patients with ulcerative colitis. The present study does not confirm their findings.

sodium This monovalent cation is absorbed both from the small and large intestines. The difference between the two is that in the small intestine the movement of sodium is largely bidirectional, absorption being balanced by secretion. Sodium transport in the colon is almost entirely a matter of absorption (Visscher, Varco, Carr, Dean, and Erickson, 1944).

Levitan, Fordtran, Burrows, and Ingelfinger (1962), using a perfusion technique, showed that the rate of sodium absorption from the human colon remained constant over a four-hour period. In the present study the amount of radioactive sodium absorbed into the blood in the normal subjects rose steadily over the first two hours and then remained constant. The two studies cannot be directly compared, because that by Levitan et al was a direct measurement of absorption whereas the present study involves an indirect assessment of colonic absorption. Conceivably, the plateau stage of our curves represents a steady state when absorption from the colon is continuing but is exactly counterbalanced by clearance from the plasma.

One of the most important findings of the present study was the much higher level of ${ }^{24} \mathrm{Na}$ in the blood of patients with ulcerative colitis than in the blood of control subjects after equal quantities of tagged sodium chloride solution had been instilled into the colon. Before deciding that this finding represents increased absorption of sodium by the colon in ulcerative colitis, two other possibilities must be considered. The first is that renal function may be impaired in ulcerative colitis with resulting slow renal clearance of absorbed sodium from the blood; the second is that the body pool of sodium may be reduced in ulcerative colitis so that absorbed sodium in the blood is equilibrating with a smaller than normal pool of sodium outside the blood. Studies of the blood levels of ${ }^{24} \mathrm{Na}$ after intravenous injection of the same quantity in patients with ulcerative colitis and control subjects show that the levels achieved are similar and the rate of clearance is likewise similar. Hence we deduce that the colon in ulcerative colitis absorbs sodium more avidly than a normal colon.

A number of physiological explanations may be offered to account for the more active absorption patterns found in ulcerative colitis. Fluid administered rectally may be propelled further up the colon in ulcerative colitis patients and so irrigate a wider area than in normal subjects (Truelove, 1956; 
Matts and Gaskell, 1961). Recently, we have studied radiologically the extent of spread of a dilute barium suspension admininstered rectally and have found no appreciable difference between ulcerative colitis patients and control subjects. We therefore judge that the extent of spread within the colon is not the salient reason for the enhanced absorption of sodium in ulcerative colitis. An alternative explanation is that the inflamed mucosa of ulcerative colitis absorbs more readily than an equal area of normal colonic mucosa. Some indirect support for this possibility comes from the findings of McInally, Campbell, Robertson, and Douglas (1952) who showed that the rate of clearance of sodium from a tissue was directly proportional to its blood supply, while Buchanan, Walls, and Williams (1954) have shown the rate of clearance to be increased in the presence of hyperaemia. The crucial factor in ulcerative colitis may be the rate at which sodium absorbed by mucosal cells from the colonic lumen is cleared into the blood as a result of the mucosal hyperaemia, but this is a possibility which remains to be examined experimentally.

In the four patients in complete clinical remission, the absorption curves for sodium were closer to those obtained from patients with active disease than to those from normal subjects. A quiescent phase in the course of ulcerative colitis can be notoriously deceptive and the reason may be that at the cellular level the mucosa still remains in an abnormal state. If this is the case, measurement of electrolyte absorption may turn out to be the best criterion for assessing cellular normality in the diseased colon although this would be a research method rather than one for routine use.

Duthie et al (1964) obtained evidence suggesting that the ability of the colon to absorb sodium is diminished in ulcerative colitis. They made their observations on patients undergoing abdominal surgery and the combined effects of anaesthesia, preoperative drugs, and direct handling of the colon may have influenced their findings.

POTASSIUM This monovalent cation is normally absorbed from the ileum and excreted by the colon (Campbell, Dickinson, and Slater, 1963), in each case in exchange for sodium.

Comparison of the colonic absorption of ${ }^{42} \mathrm{~K}$ in normal subjects and in colitis patients reveals that there is very little absorption from the colon and that it is similar in the two groups. It seems likely to be the result of a purely passive process, so that even in ulcerative colitis the cells of the mucosa retain their normal power of discrimination between electrolytes. The difference from the pattern of sodium absorption is also important because it confirms that the increased absorption of sodium from the colon in ulcerative colitis is still the result of an active process. If the increase of sodium absorption in ulcerative colitis were simply the result of increased diffusion due to partial breakdown of the mucosal membrane barrier, it would be reasonable to suppose that the level of absorption of potassium in ulcerative colitis should be similarly raised.

MAGNESIUM The principal site of magnesium absorption in man is located in the small intestine (Wacker and Vallee, 1964); the role of the human large intestine remains controversial. Graham, Ceasar, and Burgen (1960) using ${ }^{28} \mathrm{Mg}$ concluded that there was little or no absorption from the colon. Nevertheless, reports of magnesium intoxication following its administration in relatively large amounts as enemata (Fawcett and Gens, 1943; Collins and Russell, 1949; Stevens and Wolff, 1950) clearly establish that magnesium salts can be absorbed from the normal colon.

In the present study, following direct administration of ${ }^{28} \mathrm{Mg}$ into the normal colon, a small quantity was absorbed. It is possible that the capacity of the distal colon to absorb magnesium is less than that of the proximal part, as it is with sodium, chloride, and water (Levitan et al, 1962). If so, it is conceivable that there is a higher uptake from largevolume enemata which are likely to reach the proximal colon.

Hypomagnesaemia may occur in ulcerative colitis, and Thorén (1963) has recorded several such cases. In the present study the two patients with ulcerative colitis had evidence of a much greater colonic absorption of magnesium than the control subjects. The possible mechanisms for this increased uptake are likely to be the same as those suggested in the case of sodium.

CALCIUM Following the general rule that the rate of absorption of divalent cations is slower than that of the monovalent ones, calcium is absorbed very slowly, mainly from the upper part of the small intestine. There is no published record to show whether the human colon can absorb this electrolyte and the metabolic consequences of ulcerative colitis on calcium metabolism have not been investigated in detail.

In the present study, there was a small increase of radioactivity in the blood of one of the control subjects after ${ }^{47} \mathrm{Ca}$ had been introduced into the colon, showing that a little of it had been absorbed. Results obtained from the two patients with ulcerative colitis were entirely different. There was much more radioactivity in the blood of both and when 
the means of their count rates were plotted graphically, the curve showed a linear rise for three hours before beginning to flatten out (Fig. 6). The possible causes of increased sodium absorption from the colon in ulcerative colitis may apply equally to this big rise in its calcium-absorbing capacity and there may be other explanations in addition. For example, the mucosal surface of the colon becomes more alkaline in ulcerative colitis thus reducing the ionization of calcium salts and a cell membrane is less resistant to the non-ionized form of an electrolyte than it is to the ionized form.

At first sight, it is paradoxical that the colon in ulcerative colitis should be able to absorb sodium, magnesium, and calcium much more avidly than a normal colon, because it is well established that deficiencies of all these ions can occur in ulcerative colitis. Considerations other than the absorptive capacity of the colon must be taken into account to explain the heavy depletion of electrolytes which is so common in this disease. It seems to us that the crucial factor may be the volume of the faeces, which in turn may be largely dependent on the motility of the colon. If the colon rapidly passes the small-intestinal residues through itself, the sodium in those residues may be largely lost in the faeces, although some will be absorbed and rereplaced by potassium excreted by the colon in direct proportion to the amount of sodium absorbed, as there is an inverse linkage between the sodium and potassium transport systems (Koefoed-Johnsen and Ussing, 1960). The key item may be the amount of water remaining unabsorbed from the colon. If this amount is large, the total loss of electrolytes is likely to be heavy; if this large volume traverses the colon rapidly, loss of sodium will be dominant, but if it traverses less rapidly, loss of potassium will be dominant. It is conventional to regard the volume of the small-intestinal residue as being about $500 \mathrm{ml}$ per 24 hours, which would not account for the large volume of liquid faeces, often amounting to several litres, which is usual in severe ulcerative colitis. However, this conventional figure is based on the amount of ileostomy discharge in patients with well established ileostomies and there is some evidence that such patients persist in a state of chronic dehydration through deliberately limiting their fluid intake in order to keep the ileostomy discharge small, thus facilitating life with an ileostomy. A newly established ileostomy discharges a largevolume of fluid and it is possible that this resembles the output of the intact ileum more closely than does the small discharge from a long-established ileostomy. Alternatively we have to assume either that the small intestine is abnormal in ulcerative colitis and fails to absorb water efficiently, or that a large proportion of the water of the fluid stools of ulcerative colitis has been exuded from the inflamed mucosa of the colon. On clinical grounds we consider it improbable that any large proportion of the fluid in faeces in ulcerative colitis is derived by exudation, because, when we have 'uncoupled' the colon in this disease by a double-barrelled ileostomy (Truelove, Ellis, and Webster, 1965), it is unusual to have any appreciable discharge from the uncoupled colon even though it may remain intensely inflamed. Consequently we believe that most of the faecal water in ulcerative colitis must represent unabsorbed ileal efflux. This view gains some support from the recent finding by Levitan and Brudno (1967) of impaired water absorption by the colon in ulcerative colitis.

\section{SUMMARY}

Colonic absorption of sodium, potassium, magnesium, and calcium has been studied in the normal human colon and in ulcerative colitis by means of radioactive isotopes introduced per rectum in isotonic solutions.

Sodium, magnesium, and calcium were readily absorbed from the normal colon but potassium was only minimally absorbed, probably by diffusion. The blood levels of sodium, magnesium, and calcium continued to rise for two or three hours after instillation in the colon and then remained steady for the remainder of the five-hour experimental period.

In ulcerative colitis, the blood levels of sodium, magnesium, and calcium rose to much higher levels than in control subjects, but absorption of potassium remained low as in normal subjects. As far as sodium is concerned, the high blood levels in ulcerative colitis can safely be taken to represent enhanced absorption, because studies of the rate of clearance of radioactive sodium injected intravenously showed no appreciable difference between ulcerative colitis patients and control subjects.

The possible mechanisms underlying the enhanced capacity of the colon to absorb sodium, magnesium, and calcium in ulcerative colitis are discussed. The apparent paradox that depletion of these cations is not infrequent in ulcerative colitis in spite of the enhanced capacity of the colon in this disease to absorb these ions is also discussed.

We wish to thank the Wellcome Trust for making a grant to one of us (D.G.) during the period covered by this study. We also thank Glaxo Laboratories for a gift of the plastic enema bags.

\section{REFERENCES}

Buchanan, T. J., Walls, E. W., and Williams, E. S. (1954). Studies in radiosodium clearance from the skin in man. Clin. Sci., 13, 333-342. 
Campbell, E. J. M., Dickinson, C. J., and Slater, J. D. H. (1963). Clinical Physiology, 2nd ed. Blackwell, Oxford.

Collins, E. N., and Russell, P.W.(1949). Fatal magnesium poisoning following $\mathrm{MgSO}_{4}$, glycerine, water enema in primary megacolon. Cleveland Clin. Quart., 16, 162-166.

Duthie, H. L., Watts, J. M., De Dombal, F. T., and Goligher, J. C. (1964). Serum electrolytes and colonic transfer of water and electrolytes in chronic ulcerative colitis. Gastroenterology, 47, 525-530.

Fawcett, D. W., and Gens, J. P. (1943). Magnesium poisoning following an enema of epsom salt solution. J. Amer. med. Ass., 123, 1028-1029.

Graham, L. A., Caesar, J. L., and Burgen, A. S. V. (1960). Gastrointestinal absorption and excretion of $\mathbf{M g}^{28}$ in man. Metabolism, 9, 646-659.

Koefoed-Johnsen, V., and Ussing, H. H. (1960). Ion transport. In Mineral Metabolism, edited by C. L. Comar, and F. Bronner. Vol. I, Part 1A, p.169. Academic Press, New York and London.

Levitan, R. and Brudno. S. (1967). Permeability of the rectosigmoid mucosa to tritiated water in normal subjects and in patients with mild idiopathic ulcerative colitis. Gut, 8, 15-19.

-, Fordtran, J. S., Burrows, B. A., and Ingelfinger, F. J. (1962) Water and salt absorption in the human colon. J. clin. Invest., 41, 1754-1759.

Lubran, M., and McAllen, P. M. (1951). Potassium deficiency in ulcerative colitis. Quart J. Med., 20, 221-232. (New Series).

Matts, S. G. F., and Gaskell, K. H. (1961). Retrograde colonic spread of enemata in ulcerative colitis. Brit. med. J., 2, 614-616.
McInally, M., Campbell, J. A., Robertson, D. J., and Douglas, D. M. (1952). The clearance of radiosodium from the subcutaneous tissues of the leg. Clin. Sci., 11, 183-190.

Posey, E. L., and Bargen, J. A. (1950). Metabolic derangements in chronic ulcerative colitis. Gastroenterology, 16, 39-50.

Smiddy, F. G., Gregory, S. D., Smith, I. B., and Goligher, J. C. (1960). Faecal loss of fluid, electrolytes, and nitrogen in colitis before and after ileostomy. Lancet, 1, 14-19.

Stevens, R. A., Jr., and Wolff, H. G. (1950). Magnesium intoxication. Absorption from the intact gastro-intestinal tract. Arch. Neurol. Psychiat. (Chic.), 63, 749-759.

Thorén, L. (1962). Magnesium deficiency: studied in two cases of acute fulminant ulcerative colitis treated by colectomy. Acta chir. scand., 124, 134-143.

- (1963). Magnesium deficiency in gastro-intestinal fluid loss. Ibid, Suppl. 306,

Truelove, S. C. (1956). Treatment of ulcerative colitis with local hydrocortisone. Brit. med. J., 2, 1267-1272.

—, Ellis, H., and Webster, C. U. (1965). Place of a double-barrelled ileostomy in ulcerative colitis and Crohn's disease of the colon: a preliminary report. Ibid., 1, 150-153.

Visscher, M. B., Varco, R. H., Carr, C. W., Dean, R. B., and Erickson, D. (1944). Sodium ion movement between the intestinal lumen and the blood. Amer. J. Physiol., 141, 488-505.

Wacker, W. E. C. and Vallee, B. L. (1964). Magnesium. In Mineral metabolism, edited by C. L. Comar, and F. Bronner, vol. 2, Part 2A, pp. 484-521. Academic Press, New York and London.

Warner, G. T, and Oliver, R. (1962). A plastic phosphor well counter for sample volumes up to $400 \mathrm{ml}$. Brit. J. Radiol., 35, 349-352. 